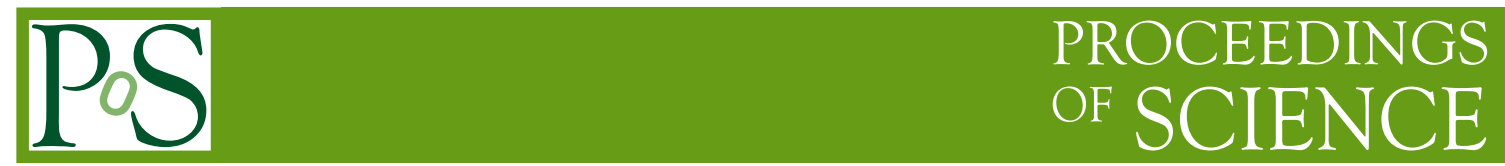

\title{
Isolated photons in deep inelastic scattering and photoproduction
}

\section{Peter J. Bussey*}

University of Glasgow, G12 8QQ, U.K.

E-mail: peter.busseyeglasgow.ac.uk

for the ZEUS Collaboration

Isolated high-energy photons have been measured in Deep Inelastic Scattering and photoproduction using the ZEUS detector at HERA, with an accompanying jet. Inclusive photon photoproduction is also measured. Cross sections are compared to theoretical predictions.

The European Physical Society Conference on High Energy Physics -EPS-HEP2013 18-24 July 2013

Stockholm, Sweden

${ }^{*}$ Speaker. 


\section{Introduction}

This talk presents measurements of isolated photon production at high transverse energy, $E_{T}$, in deep inelastic scattering (DIS) and photoproduction using the ZEUS detector at HERA. High- $E_{T}$ photons may be produced in various ways. They may be (i) produced in a hard partonic scattering process ("prompt" photons), (ii) radiated from an outgoing high- $E_{T}$ quark, or (iii) obtained as a decay product of a high energy hadron. In DIS, they may also be (iv) radiated from the incoming or outgoing lepton. In photoproduction, the processes of type (i) may be categorised as direct, in which the photon interacts in its entirety with a quark from the proton, or resolved, in which a parton contained within the photon interacts with a parton from the proton. DIS processes are predominantly direct. Processes (i) and (iv) generate outgoing photons that tend to be isolated from the other particles in the final state. A requirement that the observed photon be isolated in the event reduces the backgrounds from process (iii) and also the component of type (ii), which is not easy to calculate theoretically.

These processes are of interest both in DIS and photoproduction, since they give a distinctive perspective on QCD physics which allows particular theoretical models to be tested. In particular, the prompt photons are produced and detected directly from the basic partonic interaction and are not formed through a jet fragmentation process. In a previous publication, the ZEUS collaboration presented inclusive measurements of isolated hard photons in DIS processes [1]. Here, further measurements are presented in which a jet is observed in addition to a hard photon [2]. This enhances the prompt photon component in the data sample. In addition, preliminary results on isolated photon photoproduction are presented.

\section{Apparatus and measurement}

The ZEUS detector operated at the HERA collider, in which electrons and positrons at 27.5 $\mathrm{GeV}$ collided with protons at $920 \mathrm{GeV}$. The principal components of the ZEUS detector used in this analysis were a central drift-chamber tracker within a solenoidal magnetic field, surrounded by a uranium-scintillator calorimeter. The calorimeter was divided into three regions, forward, barrel and rear, and each region consisted of a finely segmented electromagnetic section outside which was a hadronic section with larger cells. The "forward" $(Z)$ direction is the proton beam direction.

The present DIS (photoproduction) analyses use $332 \mathrm{pb}^{-1}\left(374 \mathrm{pb}^{-1}\right)$ of data, taken during 2004-2007. A photon candidate in an event corresponds to energy deposited in a closely-spaced cluster of barrel calorimeter cells, giving a total transverse energy $E_{T}^{\gamma}$ of at least 4 (6) GeV. The pseudorapidity $\eta^{\gamma}$ of the photon candidate must lie in the range -0.7 to 0.9 , within the barrel calorimeter acceptance. Jets are reconstructed from ZEUS energy-flow objects [ [П], which combine tracking and calorimeter information, and the $k_{T}$ clustering algorithm is used [5]. The jet must have a transverse energy $E_{T}^{\text {jet }}$ of at least $2.5(4.0) \mathrm{GeV}$ and a pseudorapidity $\eta^{\text {jet }}$ in the range -1.5 to 1.8. Inclusive photoproduction events are also measured, in which a jet is not required. The photon candidate must have $\geq 90 \%$ of its energy in the electromagnetic calorimeter cells, and must be isolated in the sense that in the reconstructed jet-like object containing the photon candidate, the latter must take $\geq 90 \%$ of the transverse energy. To reduce photoproduction background in the DIS samples, the scattered beam electron (positron) must have an energy of at least $10 \mathrm{GeV}$. It must be 

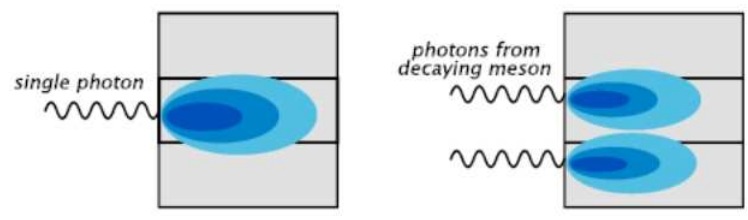

Figure 1: Schematic illustration of the showering in the ZEUS electromagnetic calorimeter of a single photon (left) and the broader distribution of a photons from a meson decay (right).

scattered at an angle of $\geq 140^{\circ}$ from the proton direction, with a transverse momentum squared, $Q^{2}$, between 10 and $350 \mathrm{GeV}^{2}$. DIS background in the photoproduction events is estimated to be small and has not been corrected for in the present preliminary results.

As outlined above, a substantial background to the prompt photon signal comes from decays of high energy neutral mesons, in particular $\pi^{0}$ mesons. To extract the photon signal, the quantity $\langle\delta z\rangle$ is used, defined as the $E_{T}$-weighted mean of the distance of the $Z$ position of the electromagnetic cells in the cluster relative to the mean $Z$ of the cluster. The broader lateral distribution of energy deposits from a meson decay is illustrated in figure 1 . The $\langle\delta z\rangle$ distribution for the entire DIS sample is shown in figure 1. It is fitted to the sum of the contribution from process (iv) (calculated), the background (freely scaled), both evaluated from the ARIADNE 4.12 Monte Carlo [6], and a freely scaled signal contribution evaluated using PYтнIA 6.416 [7]. The photon contributions have a peak at low $\langle\delta z\rangle$, plotted in units of electromagnetic calorimeter cell widths, indicating that most of the energy is found in one cell. The broader background peaks around a value of approximately 0.5 , where the cluster energy is mostly shared between two contiguous cells. Fits of this kind are performed to the data in each bin of each quantity whose cross section is to be measured, to extract the photon signal. A similar fitting procedure (without the LL contribution) is used in the photoproduction analysis, in which signal and background samples are evaluated using PYTHIA 6.416; the background is obtained using samples of simulated dijet events.

\section{ZEUS}

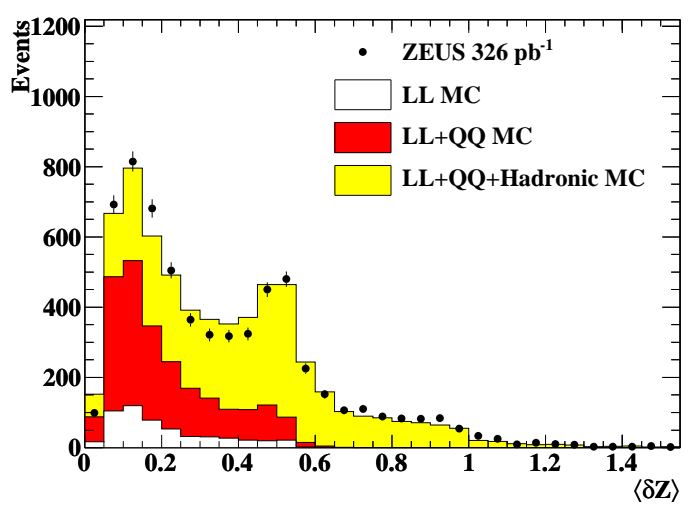

Figure 2: Distribution of the mean width $\langle\delta z\rangle$ of the electromagnetic calorimeter cell cluster forming the photon candidate in DIS processes, fitted to a combination of Monte-Carlo modelled background, a contribution of photons from process (iv) (LL), and a signal contribution from process (i) (QQ). 


\section{Results}

The resulting DIS cross sections as functions of $Q^{2}$, Bjorken $x, E_{T}^{\gamma}, \eta^{\gamma}, E_{T}^{\text {jet }}$ and $\eta^{\text {jet }}$ are shown in figure 3. Systematic uncertainties are dominated by the photon and jet energy scales, and the modelling of the background. Comparison is made with the perturbative NLO QCD model of Gehrmann-de Ridder et al. [8] and the model of Baranov et al. [9], in which unintegrated parton densities are used in a LO QCD framework. The data and the theory predictions are corrected to the hadron level. There is reasonable agreement between the experimental data and the shapes of the model distributions, but the absolute cross sections are not well predicted.

Preliminary results for the photoproduction cross sections are shown in figure 4. Inclusive photon cross sections as functions of $E_{T}^{\gamma}$ and $\eta^{\gamma}$ are followed by cross sections for a photon accompanied by a jet, as functions of $E_{T}^{\gamma}, \eta^{\gamma}, E_{T}^{\text {jet }}, \eta^{\text {jet }}$ and $x_{\gamma}^{\text {meas }}$. The quantity $x_{\gamma}^{\text {meas }}$ estimates the fraction of the incoming photon energy that is given to the observed photon+jet final state, and is defined as the sum of $\left(E-p_{z}\right)$ of the photon and the jet, divided by the sum of the same quantity over all the energy-flow objects in the event. The peak near unity is due to the direct component, which constitutes approximately half of the accepted cross section. Systematic uncertainties also include an event-modelling contribution estimated by re-evaluating the results using HERWIG 6.510 [10] instead of PythiA in the Monte-Carlo event generation.

The results are compared to the model of Fontannaz et al. [11], who perform a full NLO calculation and include contributions from box diagrams and radiation from quarks in jets. The results are also compared to the model of Lipatov et al. [12], whose calculation uses tree-level NLO terms and unintegrated parton densities in the proton only. Agreement with both models is good, but the theoretical uncertainties are substantial.

\section{References}

[1] ZEUS Collaboration, S. Chekanov et al., Measurement of isolated photon production in deep inelastic ep scattering, Phys. Lett. B 687 (2010) 16 [hep-exp/0909.4223].

[2] ZEUS preliminary result, Studies of the photoproduction of isolated photons with and without a jet at HERA, ZEUS-prel-13-001 (2011).

[3] ZEUS Collaboration, H. Abramowicz et al., Measurement of isolated photons accompanied by jets in deep inelastic ep scattering, Phys. Lett. B 715 (2012) 88 [arXiv:1206.2270].

[4] G. M. Briskin, Ph. D. Thesis, Tel Aviv University, DESY-THESIS-1998-036 (1998).

[5] S. Catani et al., Longitudinally invariant $k_{\perp}$ clustering algorithms for hadron-hadron collisions, Nucl. Phys. B 406 (1993) 187.

[6] L. Lönnblad, Ariadne version 4 - A program for simulation of QCD cascades implementing the colour dipole model, Comp. Phys. Comm, 71 (1992) 15.

[7] T. Sjöstrand et al., PYTHIA 6.4 physics and manual, JHEP 0605 (2006) 26.

[8] A. Gehrmann-de Ridder, G. Kramer and H. Spiesberger, Photon plus jet cross sections in deep inelastic ep collisions at order $O\left(\alpha^{2} \alpha_{s}\right)$, Nucl. Phys. B 578 (2000) 326 [hep-ph/ 0003082 ].

[9] S. Baranov, A. Lipatov and N. Zotov, Deep inelastic prompt photon production at HERA in the kt-factorization approach, Phys. Rev. D 81 (2010) 094034 [arXiv: 1012 . 3022]. 

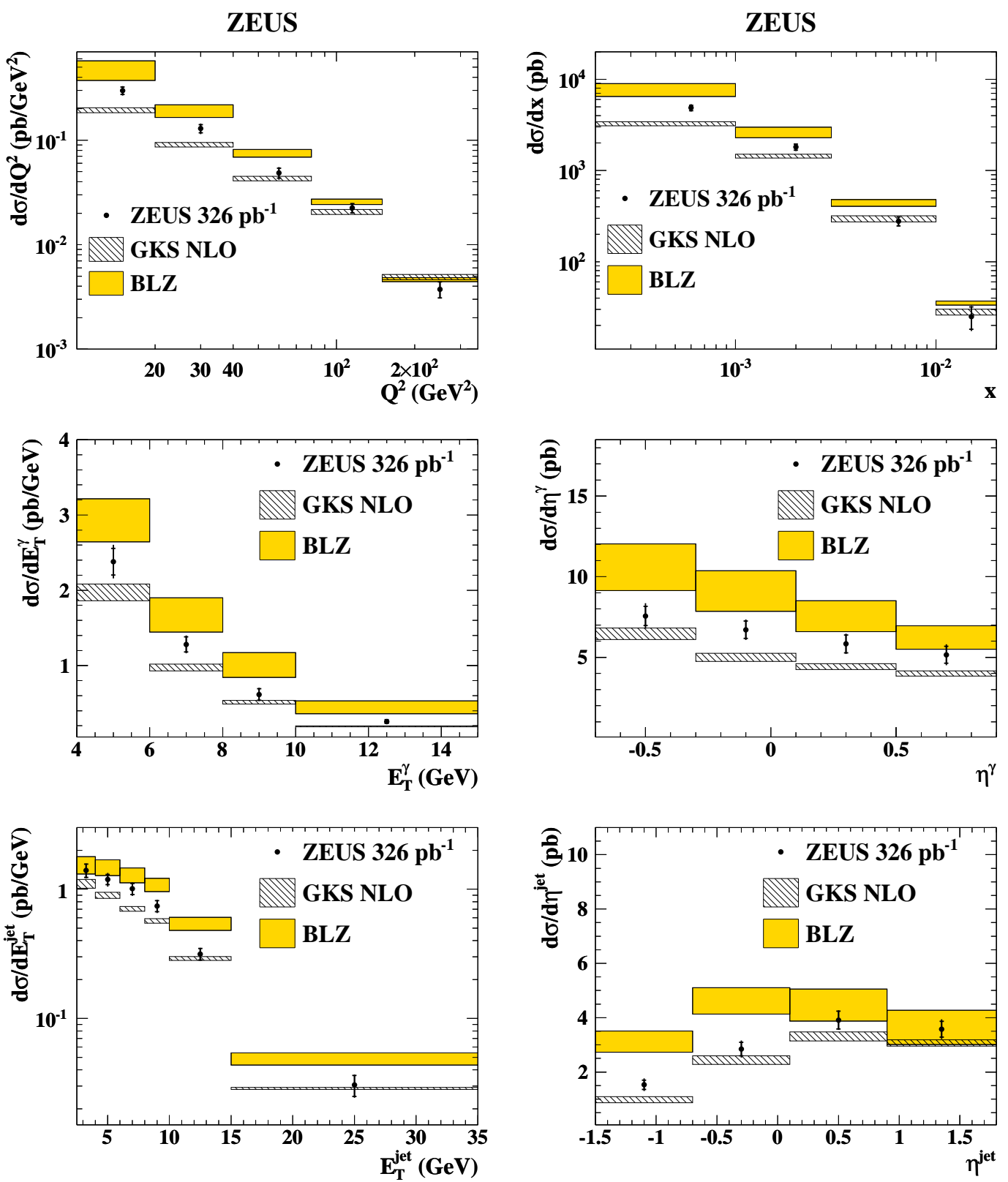

Figure 3: Cross sections for kinematic quantities in isolated photon production in DIS, as described in the text, compared to the GKS and BLZ models. The inner and outer error bars denote statistical and total uncertainties, respectively.

[10] G. Corcella et al., HERWIG 6.5: an event generator for Hadron Emission Reactions With Interfering Gluons (including supersymmetric processes), JHEP 0101 (2001) 010 [hep-ph/0011363].

[11] M. Fontannaz, J-Ph. Guillet and G. Heinrich, Isolated prompt photon photoproduction at NLO, Eur. Phys. J. 21 (2001) 303 [hep-ph/ 0105121$].$

[12] A. Lipatov, M. Malyshev and N. Zotov, An extended study of the prompt photon photoproduction at HERA with $k_{T}$-factorization, arXiv:1307.3644. 
ZEUS
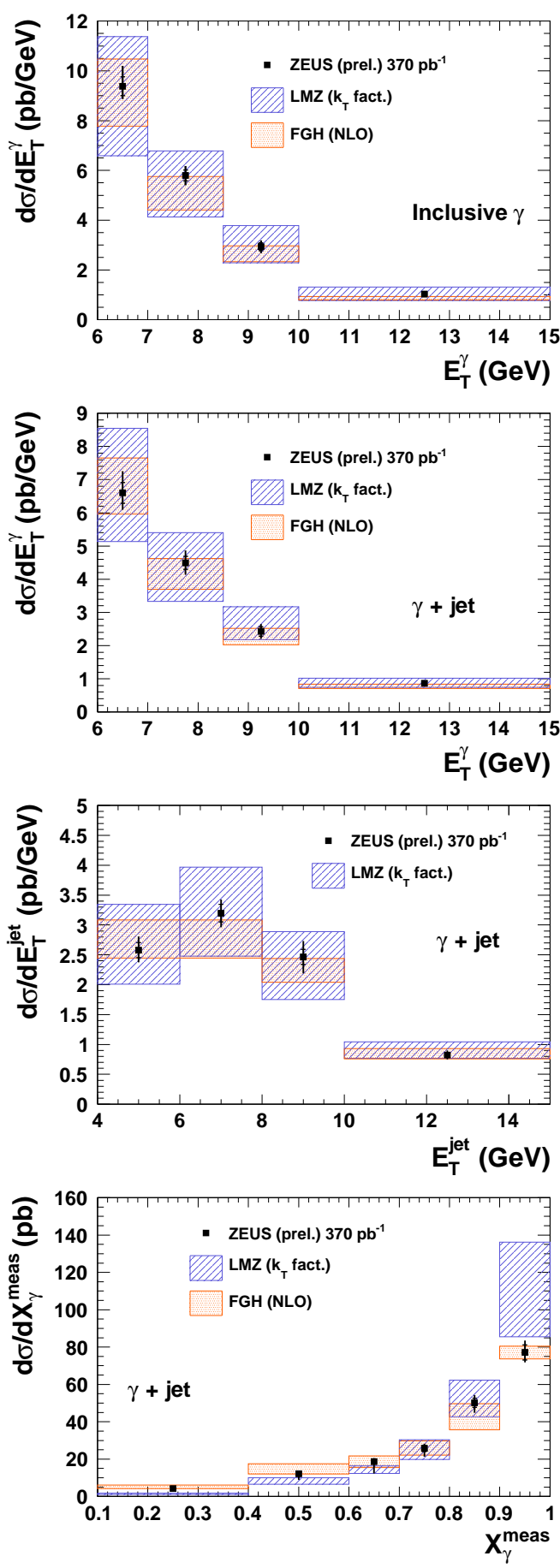

ZEUS
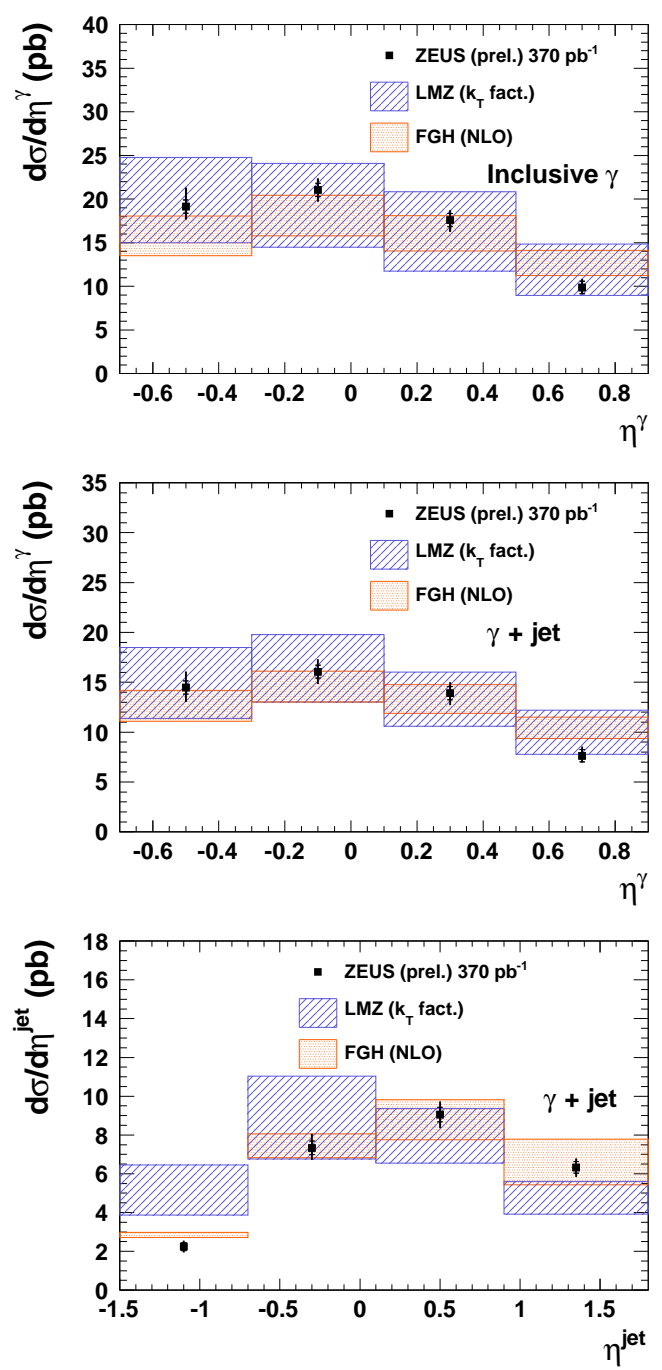

Figure 4: Cross sections for kinematic quantities in isolated photon photoproduction as described in the text, compared to the FGH and LMZ models. The inner and outer error bars denote statistical and total uncertainties, respectively. 